\title{
Novel matching lens and spherical ionizer for a cesium sputter ion source
}

\author{
D C WEISSER, N R LOBANOV, P A HAUSLADEN, L K FIFIELD, \\ H J WALLACE, S G TIMS and E G APUSHKINSKY* \\ Department of Nuclear Physics, Research School of Physical Sciences and Engineering, \\ Australian National University, Canberra, ACT 0200, Australia \\ * On leave from St. Petersburg State Technical University
}

\begin{abstract}
The beam optics of a multi-sample sputter ion source, based on the NEC MCSNICS, has been modified to accommodate cathode voltages higher than $5 \mathrm{kV}$ and dispenses with the nominal extractor. The cathode voltage in Cs sputter sources plays the role of the classical extractor accomplishing the acceleration of beam particles from $\mathrm{eV}$ to $\mathrm{keV}$ energy, minimizing space charge effects and interactions between the beam and residual gas. The higher the cathode voltage, the smaller are these contributions to the emittance growth. The higher cathode voltage also raises the Child's law limit on the Cs current resulting in substantially increased output. The incidental focusing role of the extractor is reallocated to a deceleration Einzel lens and the velocity change needed to match to the pre-acceleration tube goes to a new electrode at the tube entrance. All electrodes are large enough to ensure that the beam fills less than $30 \%$ of the aperture to minimize aberrations. The improvements are applicable to sputter sources generally.
\end{abstract}

Keywords. Beam optics; ion source; space charge.

PACS No. 29.25.Ni

\section{Introduction}

A sputter source operates by delivering neutral Cs to a hot surface where it is ionized, then accelerated in an electric field towards a sample that is sputtered by the energetic Cs ions. Some of the sample material will be ejected as negative ions that are then focused and transported for injection into a tandem accelerator. Major components of our ion source are shown schematically in figure 1 .

The primary difference between this source and higher intensity sources was the magnitude of the sputtering Cs current. Furthermore, the maximum Cs current was not limited by the flow of neutral Cs to the ionizer, but fundamentally constrained by Child's law - a quantitative statement of the fact that an ion current is limited at such a value that the electric field from the ions cancels the applied electric field extracting those ions $J=k E^{2 / 3}$, where $J$ is the limiting current and $E$ is the electric field gradient at the surface.

Our approach was therefore to investigate the electrostatics of the original source to see whether modified electric fields required to support larger Cs currents could maintain good 


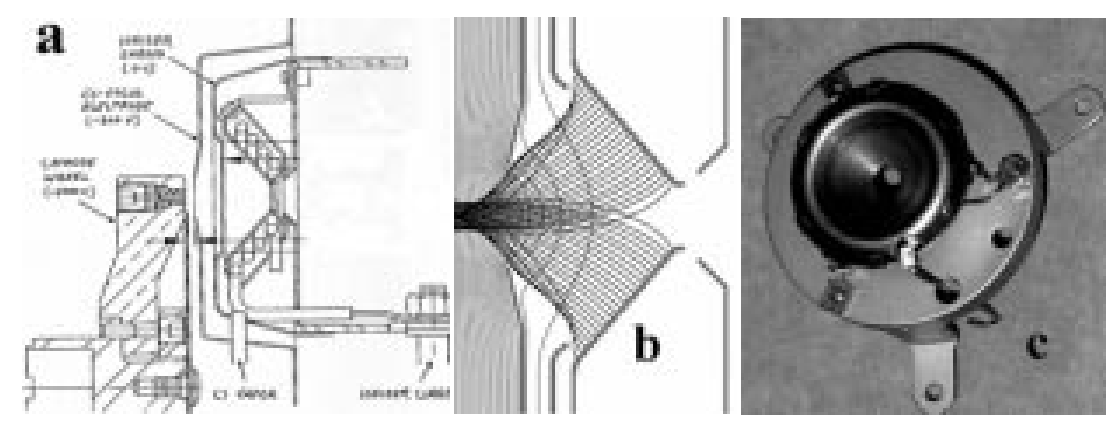

Figure 1. (a) The original geometry, (b) the $\mathrm{Cs}^{+}$trajectories and (c) the ionizer.

focus onto the sample. The calculations of electric fields and ion trajectories in the present work were performed using the code SIMION-6 [1].

\section{The original geometry}

The region of interest for calculations of potentials and ion trajectories is shown in figures $1 \mathrm{a}$ and $\mathrm{b}$. Notice that while good focus is achieved, the majority of ions initially have directions inconsistent with striking the sample (figure 1b). The observed focus is achieved by the nearly spherical bulges in the equipotentials out from the Cs-focus electrode towards the ionizer. This condition can only exist when the electric field near the surface of the ionizer is low which enforces a low Child's law current limit. For this reason, the Cs current cannot be both intense and well focused, and in this version, the source was capable of $15-20 \mu \mathrm{A}$ for ${ }^{12} \mathrm{C}^{-}$.

\section{The modified geometry}

In the modified geometry, the focusing is achieved by using a spherical ionizer, for which the initial Cs directions are consistent with converging to a point. The shape of the Csfocus electrode is then free to follow a natural equipotential imposed by the boundary conditions in its absence. Figure $2 \mathrm{~b}$ shows the calculated trajectories and equipotentials of the modified geometry, and figure $2 \mathrm{a}$, by comparison to figure $1 \mathrm{a}$, shows that the changes are possible with minimal reshaping of the ionizer and Cs-focus. Figure $2 \mathrm{c}$ is a photograph of the actual modified ionizer assembly.

There is a tightly focused $\mathrm{Cs}^{+}$beam and at the same time a large field on the ionizer surface. Despite the large $\mathrm{Cs}^{+}$currents available, the extracted ${ }^{12} \mathrm{C}^{-}$current is limited to $60-70 \mu \mathrm{A}$. This is in part because the cathode voltage is limited to $-5 \mathrm{kV}$ due to constraints of down stream optics.

Measurements of the Cs current from the modified source can be seen in figure 3. The ionizer power and Cs reservoir temperature are shown. The three curves correspond to different Cs reservoir temperatures, i.e., effectively different Cs neutral supply rates. The Cs current is limited by electric field for low potentials and limited by Cs neutral supply at high potentials. The curve for the highest reservoir temperature approaches the $E^{3 / 2}$ 


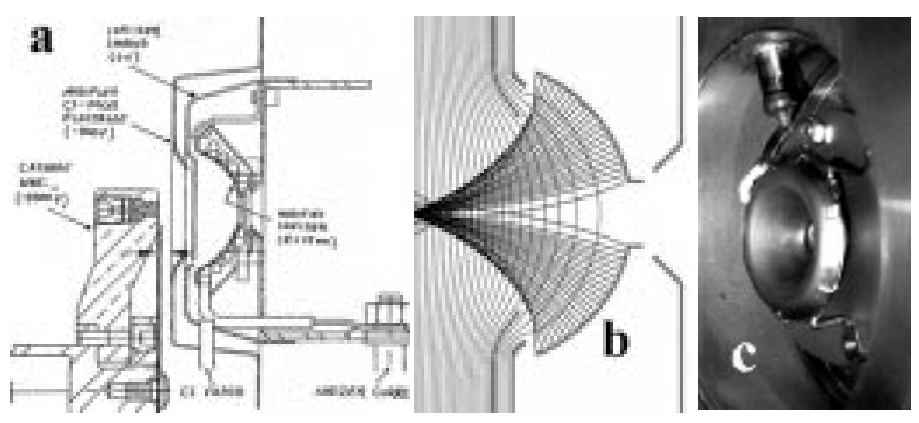

Figure 2. The modified geometry (a), the trajectories for $\mathrm{Cs}^{+}$beam in modified geometry (b) and a photograph of modified ionizer assembly (c).

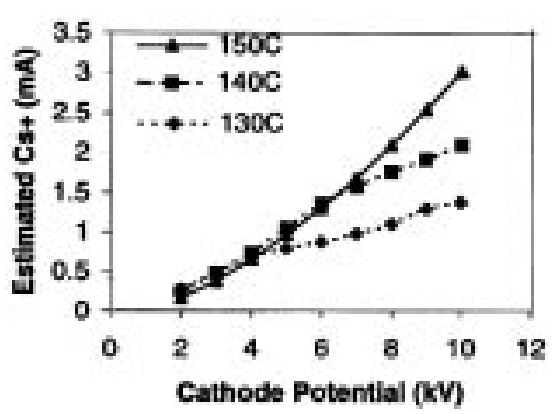

Figure 3. Estimated $\mathrm{Cs}^{+}$current for varied cathode voltage for three reservoir temperatures and fixed ionizer power of $130 \mathrm{~W}$.

dependence of Child's law throughout the range of potentials. At $5 \mathrm{kV}$ cathode potential, the Cs output is independent of the amount of $\mathrm{Cs}$ delivered, as the reservoir temperature varies from 130 to $150^{\circ} \mathrm{C}$. In contrast, at $10 \mathrm{kV}$ the additional $\mathrm{Cs}$ delivered at $150^{\circ} \mathrm{C}$ produces large beam. These $\mathrm{Cs}$ currents represent an order of magnitude improvement over the previous geometry.

Unfortunately, we cannot capture all of this improvement in the current version. The reason can be seen in figure 4, where the size of the Cs beam is reflected by the sputter erosion pattern of the samples. Note that the sputter pattern size increases markedly with increasing current, a result of the mutual repulsion among $\mathrm{Cs}^{+}$ions. To counter this, the strength of the Cs-focus must be increased but that in turn reduces the electric field on the ionizer surface thus reducing the Cs current. At present, the ${ }^{12} \mathrm{C}^{-}$output is $60-70 \mu \mathrm{A}$, consistent with an increase in Cs current, under typical running conditions, of roughly a factor of 4 .

\section{The original conventional ion beam optics}

The original ion optics comprises a single gap $15 \mathrm{kV}$ extractor, a single electrostatic lens and a $150 \mathrm{kV}$ acceleration tube and drift space. The region of interest for calculations of potentials and ion trajectories is shown in figures 5 and 6. In the SIMION calculations, the 


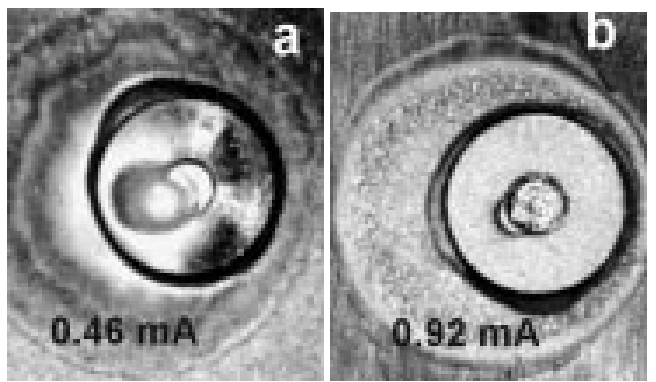

Figure 4. Photographs of sputtering for Cs-focus settings of $0.9 \mathrm{kV}$ with currents estimated as indicated.

negative current was set to $100 \mu \mathrm{A}$ covering all ion species in the beam. This provides a ${ }^{12} \mathrm{C}^{-}$current estimate of $\sim 60 \mu \mathrm{A}$ under nominal operating conditions, consistent with the measured current. The SIMION manual warns that the code does not treat space charge effects exactly. If any of the approximate space charge features of the code affect the calculated trajectories, this should be taken as a warning that space charge is important but that the code should not be relied on quantitatively. Experimental evidence that space charge effects are important in the present case is seen by the increase in the sputter pattern with Cs current as shown in figure 3c. In the original design, the exit electrode of the Einzel lens is combined with the entrance electrode of the acceleration tube. The system is intended to provide a waist in the end of the drift space - the object point of the double focusing $90^{\circ}$ magnet. This application does not require an exact image with a point-topoint reproduction of the intensity distribution at the object. What is required is a suitably small beam size at the object point, thus waist-to-waist transfer is considered rather than conjugate focusing.

The original extraction system behaves as short-focus gun. In a short-focus gun, the cathode is shielded from the extractor field by an electrode, in this case the ionizer, leading to the formation of a cross-over at very short distance beyond the extractor. This compression of the trajectories allows the space charge effects to contribute aberrations. Moreover, the ion trajectories have a very complicated dependence on the cathode voltage to extractor voltage ratio $B=V_{\text {cath }} / V_{\text {extr }}$, where $V_{\text {cath }}$ is the voltage difference between cathode and ionizer and $V_{\text {extr }}$ is the voltage applied to extractor gap, see figure 5. On the same figure, $A=\left(V_{\text {extr }}+V_{\text {cath }}-V_{\text {lens }}\right) /\left(V_{\text {extr }}+V_{\text {cath }}\right)$ is the optical strength of Einzel lens used in deceleration mode.

The ratio $B$ can have a pronounced effect on the beam transport. In the case of $B<1 / 3$, a cross-over is formed before the beam enters the Einzel lens (figure 5A). For $B>1 / 3$, the focusing effect of the extractor gap is weak resulting in no intermediate focus as shown in figure $5 \mathrm{C}$. In both cases, shown in figures $5 \mathrm{~A}$ and $5 \mathrm{C}$, the Einzel lens is filled to more than $50 \%$ of its radius, giving rise to aberrations. For $B \approx 1 / 3$ (figure $5 \mathrm{~B}$ ) the lens-filling factor is $<30 \%$ minimizing aberrations. In all cases however, a lens-filling factor of $>70 \%$ for the extractor and ionizer apertures contributes to aberrations though, because this is an accelerating lens, the safe lens-filling factor is larger.

The complicated dependence of the original extraction system performance on beam current at $B=1 / 3$ and $A=0.6$ may be seen in figure 6 . 

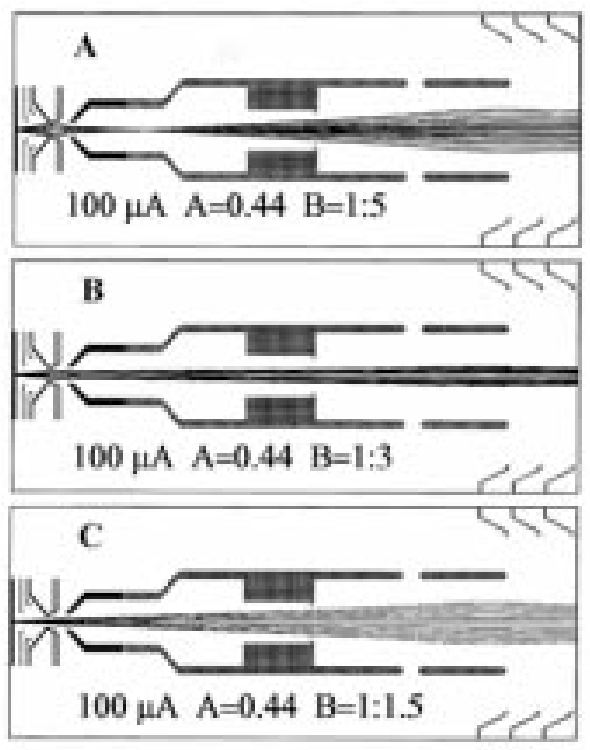

Figure 5. $100 \mu \mathrm{A}^{12} \mathrm{C}^{-}$ion trajectories in the original optics as a function of the ratio of cathode-to-extractor voltage $B=V_{\text {cath }} / V_{\text {extr. }}$ for a waist at $20 \mathrm{~cm}$ beyond the exit of the pre-acceleration tube.

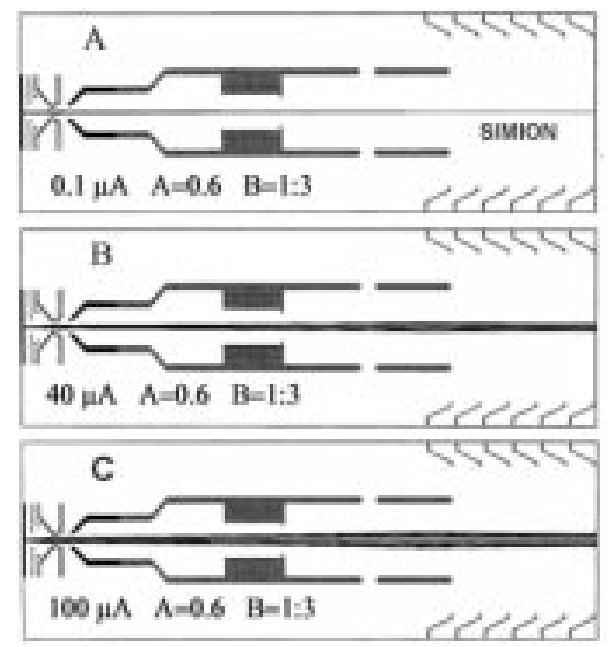

Figure 6. Ion trajectories in original optics at $B=1 / 3$ vs. beam current.

For fixed $B$, space charge effects influence the ion trajectories calculated using the ion beam repulsion option in SIMION. In this option beam repulsion is modeled by considering ions as small cylinders of charge by using their apportioned current and velocity to compute a charge per unit length. With increasing beam current, the system goes through 

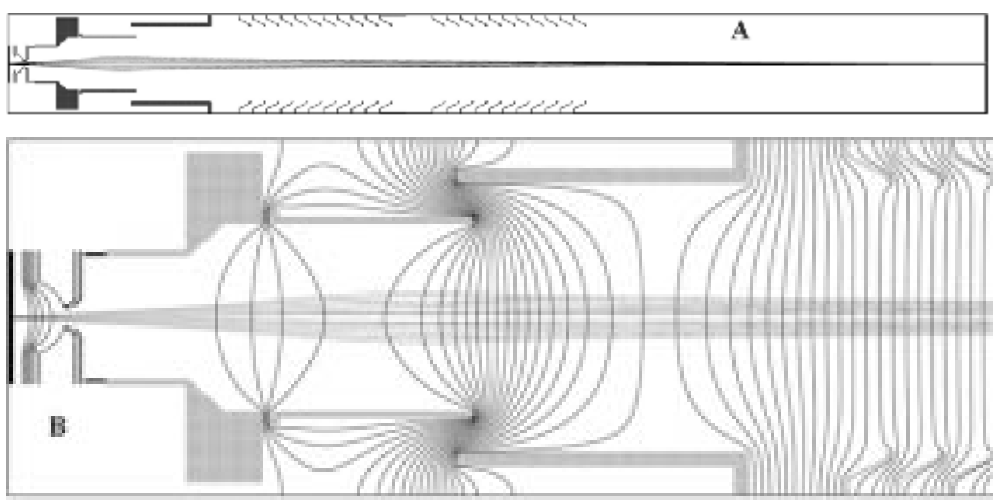

Figure 7. Trajectories for $100 \mu \mathrm{A}$ beam (A) and the equipotentials (B) to form a focus at the object slits of the inflection magnet.

a transition from forming a short focus cross-over (figure 6A) to a parallel beam (figure $6 \mathrm{~B}$ ) and then to a divergent beam after the extractor (figure 6C). To maintain a beam waste at the object slits, the Einzel needs to be reset for different beam currents. These changes in the calculated trajectories are indications of a space charge problem, not an accurate prediction of them.

To summarize this section

- The original ion optics has an optimum value for extractor-to-cathode voltage ratio $B=1 / 3$.

- A ratio of $B=1 / 3$ imposes a limit of the cathode voltage to $5 \mathrm{kV}$ for an extractor voltage of $15 \mathrm{kV}$.

- An unnecessary cross-over could contribute to space charge induced aberrations.

- Large lens-filling factors contribute to aberrations in both the extractor and Einzel.

- Trajectories have a complicated dependence on the cathode voltage to extractor voltage ratio and ion beam intensity requiring adjustment of the Einzel with beam current.

\section{The modified ion beam optics}

The design of the new optics system is based on three principles. The first is to minimize the aberrations caused by space charge by minimizing the time that the $\mathrm{Cs}^{+}$beam and the $\mathrm{C}^{-}$beams spend compacted. The second is to ensure that the decelerating electrostatic lens is filled to less than $30 \%$ of its diameter. The third is to exploit higher electric fields on the ionizer surface to raise the Child's law limit to beam intensity. In the new design shown in figures 7A and $\mathrm{B}$ these three principles are achieved and indeed, enhance one another.

The crucial requirement is to increase the cathode-to-ionizer voltage. This increases the $\mathrm{Cs}^{+}$current Child's law limit and increases the sample-sputtering rate. Space charge effects are reduced by the higher beam velocities decreasing the time that the $\mathrm{Cs}^{+}$beam spends compacted when focused on the sample, ensuring that the sputtered $\mathrm{C}^{-}$ions spend a shorter time in close proximity at the sample surface. 
One could gain the benefits of the higher $\mathrm{C}^{-}$energy by just increasing the extractor voltage although this becomes costly in insulators and power supplies. There is a more straightforward and inexpensive solution that can be explained by realizing that calling the electrode beyond the ionizer, the 'extractor', confuses its function. For there is no need to 'extract' a beam that already has 5 or more $\mathrm{keV}$ of energy. Its actual role is to focus the $\mathrm{C}^{-}$ beam and incidentally increase its energy to help match it to the accelerator tube entrance lens. These functions can be reallocated among the electrodes between the ionizer and accelerator tube.

Instead of a small diameter accelerating gap lens, we will use a large diameter decelerating Einzel lens. This ensures that the $\mathrm{C}^{-}$beam does not unnecessarily cross over before the accelerating tube that helps to avoid consequent space charge effects. The matching of the beam energy to the requirements of the entrance lens of the tube is performed by another electrode. This is both the exit electrode of the Einzel lens and the focus control electrode of the tube entrance lens. Let us call it the energy-matching electrode. The power supply for the matching electrode is the same in polarity and voltage as the previous extractor supply and the insulators are the same.

In principle, no further elements are required. For most accelerator tube gradients and cathode voltages, a matching-electrode voltage can be found to obtain the desired focus at the magnet object slits. But including a decelerating Einzel lens gains greater flexibility in the choice of gradient and cathode voltages as well as introducing the ability to adjust the magnification of the system - a zoom control. The voltage required for the Einzel is quite modest since it is only dealing with a beam at cathode voltage, though it is of opposite polarity to that used in the original optics.

Figure 7 shows the trajectories for $100 \mu \mathrm{A}$ beam (A) and the equipotentials (B) to form a focus at the object slits of the inflection magnet. Note that the equipotentials do not penetrate significantly into the small diameter exit hole in the ionizer. Since this aperture is usually filled with beam, this ensures that it contributes little to aberrations. The other two focusing electrodes are also not filled beyond $30 \%$ in radius also ensuring small aberrations from them. This will increase the magnification of the system but still place the waist at the object slits.

\section{Comparison studies of original and modified beam optics}

Since the combination of lenses is complex and the effect of space charge not easily simulated, the comparison of aberrations from the old and new optics is not straightforward. One measure of the aberrations is given by the diameter of the disk of least confusion $D$, - the smallest spot formed by the lens system. In the original beam optics, there are two major effects responsible for the minimal $D$ value, namely, the spherical aberration $C_{\mathrm{s}}$ of the combination of lenses and space charge. The diameter $D_{\mathrm{s}}$ due to spherical aberrations, is related to the angular convergence of the beam in the drift space $D_{\mathrm{s}}=C_{\mathrm{s}} \theta^{3} / 2$ [2]. The minimal value of $D_{\mathrm{c}}$ caused by space charge effects is given by $D_{\mathrm{c}}=D_{0} \exp \left(-\theta^{2} / 2 K\right)$, where $D_{0}$ is the maximum diameter of the beam and $K$ is the perveance of the beam. For ${ }^{12} \mathrm{C}^{-}$beam $K=26.3 \times 10^{5} I / E^{3 / 2}$ [2], where $I$ is the beam current and $E$ is the beam energy. If both effects are present, the minimal value of disk of least confusion caused by spherical aberration and space charge effects $D_{\text {sc }}$ is given by

$$
D_{\mathrm{sc}} \approx\left(D_{\mathrm{s}}^{2}+D_{\mathrm{c}}^{2}\right)^{1 / 2} .
$$




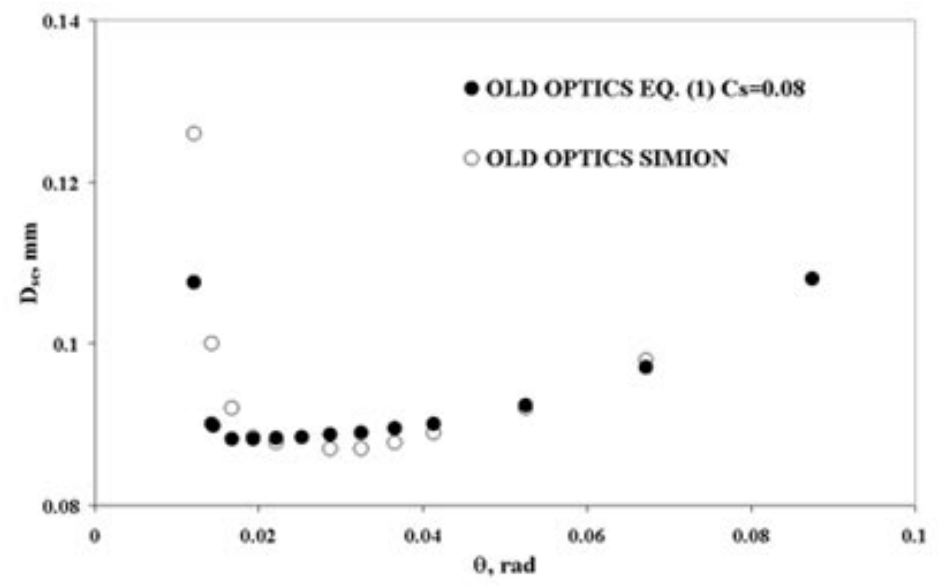

Figure 8. Calculated values of $D_{\mathrm{sc}}$ vs. angular convergence of the beam in the drift space $\theta$ for the old optics.

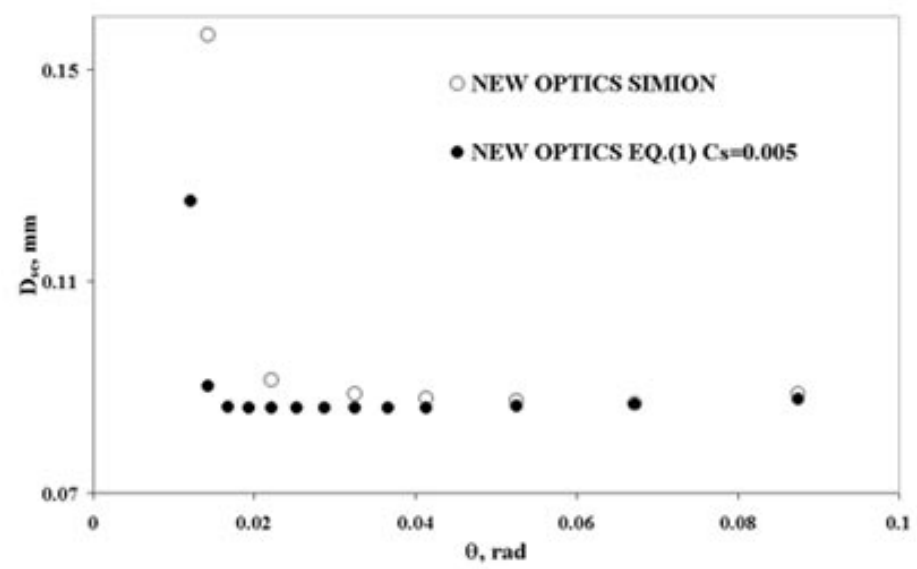

Figure 9. Calculated values of $D_{\mathrm{sc}}$ vs. angular convergence of the beam in the drift space $\theta$ for the new optics.

Calculated values of $D_{\mathrm{sc}}$ vs. angular convergence $\theta$ for the old and new optics using SIMION and Lawson formula (1) can be seen in figures 8 and 9 respectively. Values of $C_{\mathrm{s}}$ and $E$ were varied to achieve the best fit of eq. (1) to the SIMION calculation. The beam current was set to $100 \mu \mathrm{A}$ and $D_{0}$ to $12 \mathrm{~mm}$.

The rise of the curves in figures 8 and 9 with increasing $\theta$ depends on the value of aberration coefficient $C_{\mathrm{s}}$. The aberration coefficient $C_{\mathrm{s}}$, of the new optics is 16 times smaller than that for the conventional design up to the exit waist from the pre-accelerating tube for $100 \mu \mathrm{A}$ of the beam. These aberrations distort the phase space ellipse while retaining its original area. For the purpose of beam transport, the area of ellipse that can enclose all the distorted phase space is important. This is illustrated in figure 10 where the higher aberrations in old system raises the effective beam emittance. 

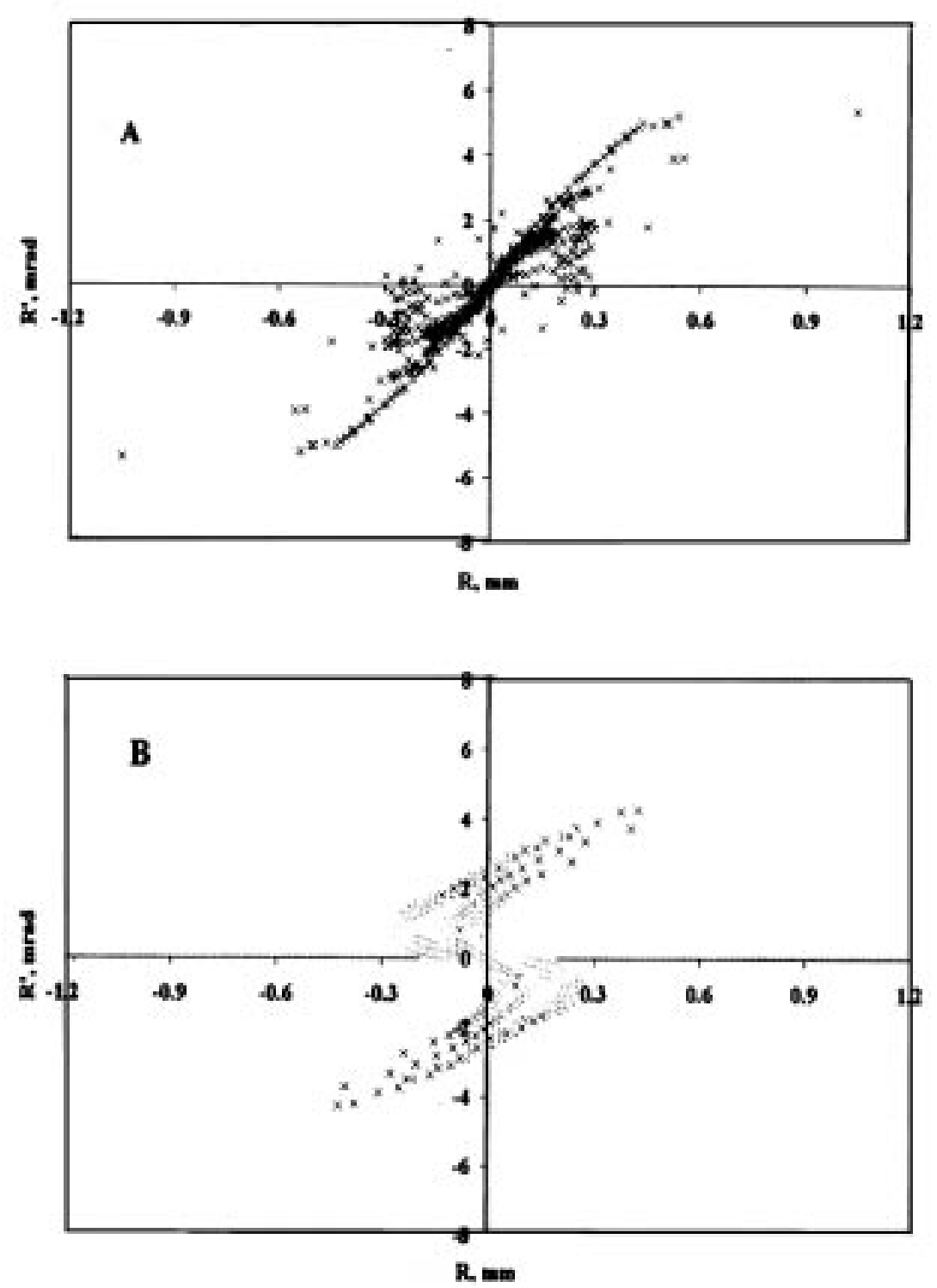

Figure 10. Emittance patterns of the beams generated by old (A) and new (B) optics, calculated for a plane $1.3 \mathrm{~m}$ downstream of the cathode by the code SIMION. The effective emittance growth is caused by spherical aberrations and space charge effects.

Statistical normalized (rms) emittance was calculated using equation $\varepsilon_{\mathrm{rmsn}}=$ $\beta \gamma\left(\left\langle R^{2}\right\rangle\left\langle R^{\prime 2}\right\rangle-\left\langle R R^{\prime}\right\rangle^{2}\right)^{1 / 2}$, where $\beta=v / c$ and $\gamma=\left(1-\beta^{2}\right)^{-1 / 2}$ [2]. Application of this equation leads to a $0.7 \pi \cdot \mathrm{mm} \cdot \mathrm{mrad}$ normalized emittance for old system while the value of $0.45 \pi \cdot \mathrm{mm} \cdot \mathrm{mrad}$ is derived for the new system. 


\section{Future efforts}

While the changes implemented in the present work were extremely successful, directions for future effort are clear. Further calculations of ion trajectories including the effects of space charge will permit adequate focusing of even larger Cs currents. In addition, making the ion source and extraction optics compatible with cathode voltages over the present 5 $\mathrm{kV}$ will yield obvious gains in intensity.

\section{References}

[1] David A Dahl, 43rd ASMS conference on mass spectrometry and allied topics, 21-26 May 1995, Atlanta, Georgia, p. 717.

[2] J D Lawson, The physics of charged-particle beams, Oxford, 1977 